\title{
La resiliencia como factor protector del cuidador informal en la labor del cuidado: Una revisión sistemática cualitativa en salud
}

\author{
Irene Soto-Fernández ${ }^{1,2}$, Patricia Domínguez-Isabel ${ }^{1,3}$, Blanca Espina \\ Jerez $^{1,4}$, Maria Jesus Bocos Reglero ${ }^{1,5}$, Maria Angustias Torres Alaminos ${ }^{1,}$ \\ 5, Sagrario Gómez-Cantarino ${ }^{1,5}$ \\ ${ }^{1}$ Estudio asociado HISAG-EP, Proyecto estructurante Historia y Epistemología, UICISA:E, \\ Coimbra, Portugal. \\ ${ }^{2}$ Escuela Pública, Ciudad de Nara. Avenida Francia 8, 45005, Toledo, España. Enfermera \\ Escolar, irene.soto.due@hotmail.com I https://orcid.org/0000-0002-1140-9544 \\ ${ }^{3}$ Hospital Virgen de la Salud, Toledo. Servicio de Urgencias, Sescam, España I \\ patriventas98@hotmail.com I https://orcid.org/0000-0001-6894-2270 \\ ${ }^{4}$ Psicologa, Universidad Complutense, Máster en Psicologia Clínica, España I \\ b.espinajerez@gmail.com I https://orcid.org/0000-0001-9961-072X \\ ${ }^{5}$ Universidad de Castilla-La Mancha - Campus de Toledo, Departamento de Enfermería, \\ Fisioterapia y Terapia Ocupacional, Toledo. 45071, España I chusmatin@yahoo.es; \\ angustiast@gmail.com; Sagrario.Gomez@uclm.es I https://orcid.org/0000-0003-3288-7796; \\ https://orcid.org/0000-0002-4523-5634; https://orcid.org/0000-0002-9640-0409
}

Resumen: Introducción: El envejecimiento puede generar una situación de dependencia creando la necesidad de cuidados atendidos por cuidadores/as informales, figuras importantes para satisfacer las necesidades básicas de esa población mayor. Los cuidadores/as informales son relevantes para la sociedad por su labor en referencia al cuidado. Por tanto, es importante incidir en su resiliencia como factor protector asociado al cuidado y reforzarlo con políticas sociales. Objetivo: Realizar una revisión sistemática para analizar la resiliencia como factor protector en la salud del cuidador/a informal que está al cuidado de personas mayores dependientes. Métodos: Se realizó una revisión sistemática desde el enfoque PRISMA y el método PICO. Se llevó a cabo una búsqueda bibliográfica desde marzo a enero de 2021 en las bases de datos de Pubmed, Web of Science y SciELO. Se revisaron sistemáticamente los artículos científicos relacionados con la resiliencia asociados a la salud del cuidador/a informal en base a una serie de criterios de inclusión. Se utilizaron descriptores DeCS y MesH con el operador booleano "AND" y "OR" y palabras clave como "cuidado", "cuidador informal", "dependencia", "envejecimiento" y "resiliencia". Resultados: Inicialmente se han encontrado 936 estudios. Una vez aplicados los criterios de inclusión la muestra ha quedado conformada por 9 artículos científicos. La resiliencia es un factor protector asociada a la salud del cuidador informal que conlleva un aumento de la calidad de vida tanto física como mental. Conclusiones. Cuidar a quien cuida es importante para que la sociedad se vea indirectamente reforzada en esta ardua tarea. Las investigaciones sobre la resiliencia asociadas al cuidado son de vital interés a todos los niveles (social, económico, particular) y se puede considerar como una estrategia social a la hora de proteger la salud y el bienestar del cuidador/a informal.

Palabras clave. Cuidados Informales; Dependencia; Envejecimiento; Resiliencia y Salud.

Resilience as a Protective Factor for Informal Caregivers in Caregiving: A Systematic Review

Abstract: Introduction: Ageing can generate a situation of dependency, creating the need for care provided by informal carers, who are important figures in meeting the basic needs of this older population. Informal caregivers are relevant to society because of their care work Therefore, it is important to focus on their resilience as a protective factor associated with caregiving and to reinforce it with social policies. Objective: To conduct a systematic review to analyse resilience as a protective factor in the health of informal caregivers caring for dependent elderly people.Methods: A systematic review was carried out using the PRISMA approach and the PICO method. A literature search was carried out from March to January 2021 in Pubmed, Web of Science and SCIELO databases. Scientific articles related to resilience associated with informal caregiver health were systematically reviewed based on a set of inclusion criteria. DeCS and MesH descriptors were used with the Boolean operator "AND" and "OR" and keywords such as "care", "informal caregiver", "dependence", "ageing" and "resilience". Results. Initially 936 studies were found. Once the inclusion criteria had been applied, the sample consisted of 9 scientific articles. Resilience is a protective factor associated with the health of the informal caregiver that leads to an increase in both physical and mental quality of life.Conclusions: Caring for the caregiver is important for society to be indirectly strengthened in this arduous task. Research on resilience associated with caregiving is of vital interest at all levels (social, economic, particular) and can be considered as a social strategy to protect the health and well-being of the informal caregiver.

Keywords. Ageing; Dependency; Health; Informal caregiver; Resilience. 


\section{Introducción}

Los avances científicos han proporcionado un aumento de la esperanza de vida de las personas en todo el mundo y un mayor bienestar, alargando en consecuencia la edad de la muerte. Sin embargo, estos avances no han podido erradicar las enfermedades crónicas generadoras de una necesidad en los cuidados de las personas mayores que las sufren (Schmidt, 2016). Por ello, el envejecimiento y la dependencia son un tándem inseparable, especialmente a partir de los 85 años (Whitbourne \& Whitbourne, 2012). La enfermedad en la persona mayor conforma una situación de dependencia y este hecho augura que un familiar cargará con los cuidados de esta persona (Pereira \& Soares, 2015). Esta situación genera una dependencia para el cuidado y ese cuidado lo lleva a cabo un cuidador/a, y si el cuidado se da en el ámbito familiar, el cuidador/a recibe el nombre de cuidador/a familiar o informal.

Ya en 1969, Zarit apuntó que normalmente el cuidado en el hogar de una persona dependiente es asumido por un familiar. Cuando se origina una situación de dependencia dentro de un hogar, se da una reestructuración de la familia por el cuidado de ese familiar pero no es meditada sino que viene en cierto modo impuesta por el nivel socioeconómico y la disponibilidad del cuidador/a para el cuidado en el momento que se origina la necesidad (Hedler, 2016). Además, las políticas sociales de los países europeos tienen conciencia de que la persona mayor prefiere envejecer en su hogar y, para ello, fomentan programas de cuidados de larga duración en los domicilios con el fin de complacer dicho propósito (OCDE, 2015).

La labor de cuidar a personas en situación de dependencia es un recurso muy valioso para la sociedad y genera un ahorro importante en las arcas estatales (Banchero \& Mihoff, 2017). En este sentido, se asume por un familiar un servicio no remunerado que si lo proporcionara el estado sería muy costoso económicamente. Por otra parte, es un recurso limitado y cada vez más escaso ya que el tener menos hijos en la actualidad disminuye los potenciales cuidadores/as informales del futuro (Banchero \& Mihoff 2017).

El cuidador/a informal de persona dependiente tiene sentimientos ambivalentes con respecto al cuidado. Por un lado, presenta algunas nociones negativas del cuidado (Banchero \& Mihoff 2017), pero por otro, no se debe olvidar que cuidar también tiene una serie de consecuencias positivas (Machado, 2020).

En definitiva, existen múltiples formas de abordar el cuidado desde una perspectiva positiva trayendo múltiples beneficios para las partes implicadas, entre ellas se encuentra la resiliencia del cuidador/a informal.

La resiliencia se definió por Ferreira en el 1975 como extensión física de un cuerpo ante una adversidad y la posterior vuelta a su ser evitando la deformidad. Más adelante, en 2001 el concepto fue pulido por Lindström, quien llamó resiliencia al proceso de superación ante momentos difíciles con el objetivo de aprender de lo vivido y manejar habilidades para afrontar mejor la vida, es decir, un afán de superación continuo individual ante las adversidades del día a día de cada persona. Es un mecanismo de protección natural del individuo para disminuir los posibles factores de riesgo que le van surgiendo a lo largo de su vida. Pesce (2005) define cuatro factores de protección de la resiliencia y pretende dar las herramientas necesarias para afrontar una situación de estrés y generar resiliencia en el individuo.

Si se extrapola la resiliencia a la tarea de cuidar, Pinheiro (2004) afirma que tanto las personas mayores que realizan la labor del cuidado como la persona en situación de dependencia, a pesar de estar en una difícil situación tienen una predisposición a la positividad y así crean su bienestar, de esta manera se llega a un envejecimiento con éxito y la labor de cuidar es más fácil. Por otro lado, Pessotti (2018) y Trapp (2015) señalan la correlación positiva existente entre la calidad de vida del cuidador y la resiliencia, corroborando que los cuidadores con más resiliencia tienen mayor calidad de vida percibida, son más religiosos y tienen menores síntomas depresivos (Pessotti, 2018). 
Por ello, la resiliencia parece ser una fortaleza personal para el cuidado asociado a la calidad de vida física y mental del cuidador/a informal (Trapp, 2015), significando un óptimo instrumento en el cuidado que predispone al positivismo en el cuidador/a informal.

Otros autores como Araújo y Faro (2016) dan nociones para mantener la resiliencia, en ellas están: tener una dieta sana, crear endorfinas (risa, deporte, sueño...), guiar las emociones, gobernar los impulsos, ser optimista, examinar el entorno, empatizar con los demás, ser autoeficaces, tener disciplina y tener confianza en sí mismo (Araújo \& Faro 2016). De este estudio se extrae que si el cuidador/a informal cumple con los apartados expuestos anteriormente, se genera una mayor resiliencia y esto revertirá en su bienestar y finalmente en la persona cuidada.

Quero (2005), apunta que la formación de las personas conforma un factor protector ante las adversidades de la vida, es decir, ellas tienen una mayor resiliencia. También ocurre lo mismo con el ejercicio físico, las personas que lo practican de forma habitual tienen un mejor ánimo y entrenan una de las habilidades que le conforman mayor resiliencia (Araújo \& Faro 2016).

Por todo lo anterior, es importante capacitar al cuidador/a informal en la labor de cuidar para darle las habilidades necesarias y potenciar su resiliencia (Pinheiro, 2004), ya que aumentará la capacidad del cuidador/a para llevar en mejores garantías la difícil tarea del cuidado (Martínez-Linares, 2020).

\section{Metodología}

A continuación, se detalla la metodología que se ha utilizado para conformar la revisión sistemática referida al factor protector de la resiliencia en la salud del cuidador/a informal de personas mayores en situación de dependencia.

\subsection{Diseño}

Se ha llevado a cabo una revisión sistemática siguiendo la guía de Preferred Reporting Items for Systematic Reviews and Meta-Analyses (PRISMA) (Beller, 2013), realizando una búsqueda exhaustiva en las siguientes bases de datos: PubMed, Web of Science y SciELO. Los artículos analizados corresponden a la franja temporal comprendida entre los años 2015 a 2020. Los resultados de la investigación se sintetizaron mediante estrategias que evitan el sesgo y el error aleatorio. Estas estrategias incluyeron la clasificación sistemática de todos los artículos potencialmente relevantes y la descripción del diseño metodológico. También el análisis y la extracción de información de los mismos, así como la presentación e interpretación de los resultados.

La búsqueda se realizó desde el 01 marzo de 2020 hasta el 31 enero de 2021. Para iniciar la revisión sistemática y poner en marcha el motor de búsqueda se formuló la pregunta de investigación diseñada a través de la metodología PICO (Gálvez, 2004 \& Santillán, 2018), estructurando los datos de la manera siguiente:

P: (Paciente o problema de interés), el cuidador/a informal que cuida a personas mayores dependientes.

I: (Intervención), observación del factor protector en la salud del cuidador/a informal.

C: (Comparación), personas mayores que no se encuentran en una situación de dependencia.

O: (Outcomes) la resiliencia. 
Vol. 8 | Investigación Cualitativa en Salud: Avances y Desafíos

Por ello se formula la siguiente pregunta de investigación: ¿Es la resiliencia un factor de protección en la salud del cuidador/a informal de personas mayores en situación de dependencia? Siendo una cuestión clara y directa que protegerá de posibles sesgos.

\subsection{Criterios de Selección}

\subsubsection{Criterios de Inclusión}

Como se observa en la Figura 1, los documentos recuperados durante las búsquedas se verificaron con los siguientes criterios de inclusión: (1) informe original a texto completo publicado; (2) artículos que incluyen la perspectiva de los cuidados familiares de personas dependientes mayores de 60 años; (3) estudios que indican las experiencias y las necesidades de los cuidadores/as informales en los cuidados de personas mayores; (4) artículos publicados en un periodo anterior a 5 años previos a la revisión sistemática; (5) artículos escritos en inglés, portugués o español.

\section{Criterios de inclusión}

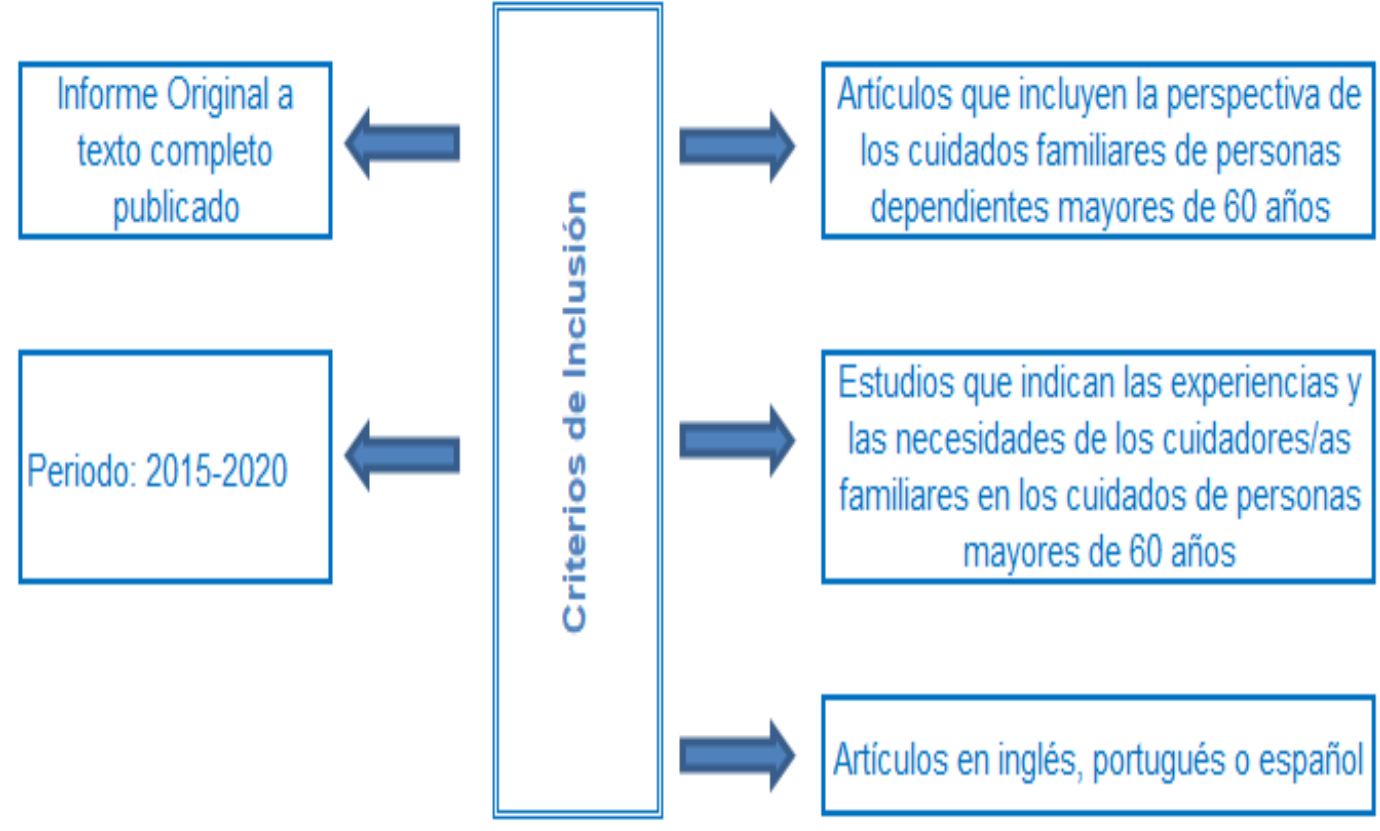

Fig. 1. Nota: elaboración propia.

\subsubsection{Criterios de Exclusión}

Los documentos desechados durante la búsqueda se verificación con los siguientes criterios de exclusión: (1) artículos sin acceso libre, (2) estudios sobre los cuidados formales, (3) investigaciones realizadas en el ámbito institucional (socio sanitario, residencias de mayores), (4) artículos en donde no se encontrasen en una situación de dependencia y (5) artículos de revisión sistemática. Se detalla un resumen a continuación en la Figura 2. 


\section{Criterios de exclusión}

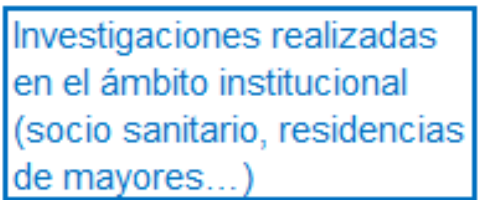

Articulos en donde las

personas cuidadas fueron

menores de 60 años y no

se encontraban en situación

de dependencia
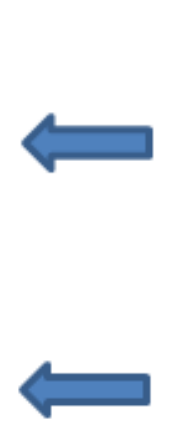

Artículos de revisión

sistemática

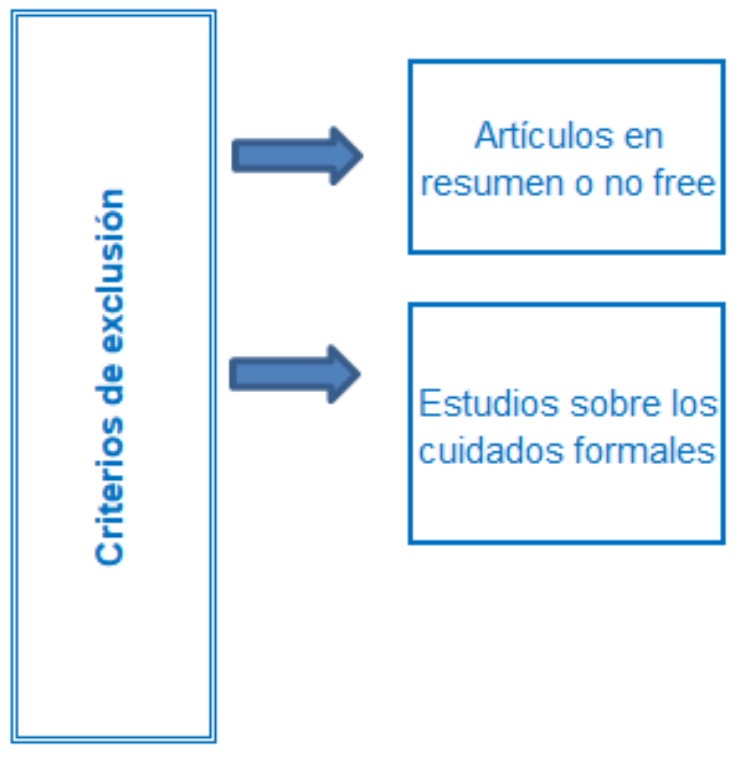

Fig 2. Nota: Elaboración propia.

\subsection{Estrategias de Búsqueda de la Literatura Científica}

Esta investigación se ha llevado a cabo por la emergente dificultad de incluir estudios que reflejaran la experiencia positiva en los cuidados del cuidador/a informal, los factores de protección asociados al cuidado y la disposición de la sociedad ante ello. También se debió a la necesidad de incorporar estudios que abarcaran la perspectiva de la humanización de la atención en el ámbito del hogar en la figura del cuidador/a informal. En este tipo de estudio se han incluido diseños cualitativos a través de una revisión sistemática de la literaria científica.

Las fuentes de datos consultadas fueron las que se describen a continuación:

-PubMed es un impulsor de búsqueda de acceso libre y permite consultar los materiales de la base de datos Medical Literature Analysis and Retrieval System Online (MEDLINE)

-Web of Science es un servicio en línea de información científica, suministrado por Clarivate Analystics, integrado en ISI Web of Knowledge, Wok. Creada por Eugene Garfield y fundada en 2016, aunque su fecha de lanzamiento fue en 1997.

-Scientific Electronic Library Online (SciELO) es un modelo para la publicación de revistas en internet. Su fecha de lanzamiento es 1997.

Las fuentes elegidas fueron principalmente primarias. Se usaron tanto artículos científicos como informes ministeriales, dictámenes o estadísticas. La literatura científica se buscó en la lengua de origen documental y por ello, hay articulado en inglés, portugués y español.

La terminología tesauros elegida con DeSH son: "cuidado familiar", "dependencia psicológica", "envejecimiento", "resiliencia psicológica y "salud" y con Medical Subject Headings (MeSH): "ageing" "dependency psychological”, "Health", "informal caregiver" and "resilience phycological". Además las palabras clave seleccionadas se han enlazado entre sí con los siguientes operadores booleanos AND y OR para obtener unos resultados de búsqueda más completa.

Una vez formulada la búsqueda con las palabras clave y los operadores booleanos empleados, se pueden observar los resultados obtenidos en el Tabla 1. Finalmente, el resultado del proceso de búsqueda de la revisión sistemática han sido nueve artículos. 
Tabla 1. Búsqueda de artículos

\begin{tabular}{|c|c|c|c|c|}
\hline $\begin{array}{l}\text { Base de } \\
\text { datos }\end{array}$ & $\begin{array}{l}\text { Total de la } \\
\text { Búsqueda }\end{array}$ & $\begin{array}{l}\text { Palabras } \\
\text { clave }\end{array}$ & $\begin{array}{l}\text { Artículos } \\
\text { seleccion } \\
\text { ados }\end{array}$ & Titulo del articulo \\
\hline \multirow{6}{*}{$\begin{array}{l}\text { Pub } \\
\text { Med }\end{array}$} & \multirow{6}{*}{785} & \multirow{6}{*}{$\begin{array}{c}\text { Cuidado } \\
\text { informal, } \\
\text { Dependencia, } \\
\text { Envejecimiento, } \\
\text { Salud, } \\
\text { Resiliencia }\end{array}$} & \multirow{6}{*}{6} & $\begin{array}{l}\text { 1.En sus propias palabras: cómo los } \\
\text { cuidadores familiares de personas con } \\
\text { demencia entienden la resiliencia }\end{array}$ \\
\hline & & & & $\begin{array}{l}\text { 2.Adapting the Resilience Framework for } \\
\text { Family Caregivers of Hospice Patients With } \\
\text { Dementia }\end{array}$ \\
\hline & & & & $\begin{array}{l}\text { 3.Family Dynamics and Personal Strengths } \\
\text { among Dementia Caregivers in Argentina }\end{array}$ \\
\hline & & & & $\begin{array}{l}\text { 4.Mediating effect of social support on the } \\
\text { relationship between resilience and burden } \\
\text { in caregivers of people with dementia }\end{array}$ \\
\hline & & & & $\begin{array}{l}\text { 5.Family caregivers of elderly with dementia } \\
\text { Relationship between religiosity, resilience, } \\
\text { quality of life and burden }\end{array}$ \\
\hline & & & & $\begin{array}{l}\text { 6.Personal strengths and health related } \\
\text { quality of life in dementia caregivers from } \\
\text { Latin America }\end{array}$ \\
\hline & & $\begin{array}{l}\text { Cuidado } \\
\text { informal, }\end{array}$ & & $\begin{array}{l}\text { 1.Psychometric properties of a resilience } \\
\text { scale in family caregivers of older adults }\end{array}$ \\
\hline $\begin{array}{l}\text { Web of } \\
\text { Science }\end{array}$ & 144 & $\begin{array}{l}\text { Dependencia,En } \\
\text { vejecimiento, } \\
\text { Salud, } \\
\text { Resiliencia }\end{array}$ & 2 & $\begin{array}{l}\text { 2.Implementación del programa de } \\
\text { intervención psicológica en resiliencia para } \\
\text { cuidadores informales de pacientes con } \\
\text { demencia tipo Alzheimer PIRCA }\end{array}$ \\
\hline SCIELO & 7 & $\begin{array}{c}\text { Cuidado } \\
\text { informal, } \\
\text { Dependencia,En } \\
\text { vejecimiento, } \\
\text { Salud, } \\
\text { Resiliencia }\end{array}$ & 1 & $\begin{array}{l}\text { 1.Caregivers' resilience is independent from } \\
\text { the clinical symptoms of dementia }\end{array}$ \\
\hline
\end{tabular}

\subsection{Proceso de Identificación y Selección de Estudios}

En la Figura 3 se presenta el diagrama de flujo que configura el proceso de selección de los artículos científicos de la revisión sistemática bajo el modelo PRISMA. Se obtuvieron un total de 936 registros iniciales, siendo Pubmed la base de datos en los que se obtuvo un mayor registro de trabajos que cumpliesen los criterios de inclusión del estudio. Posteriormente se procedió a eliminar los artículos duplicados, reduciendo considerablemente el número de artículos encontrados $(n=794)$. A este número de reportes encontrados, se aplicó el filtro de la accesibilidad de los textos y el periodo temporal, obteniendo un total de artículos resultantes $(n=106)$. Posteriormente, se rechazaron aquellos que no cumplían con los objetivos propuestos $(n=52)$, y/o con la pregunta de investigación, el número resultante fue $n=54$. Se escogieron 29 artículos para leer a texto completo por ceñirse a los objetivos planteados en la revisión sistemática. Una vez analizado el contenido de dichos artículos científicos de forma pormenorizada y, una vez se comprobó que si cumplían los criterios de inclusión y exclusión se seleccionaron para el trabajo un total 9 estudios de investigación. La muestra de artículos seleccionados recoge datos y análisis de diferentes lugares a nivel mundial y ha quedado formado por los siguientes trabajos: Trapp et al. (2015), Dias et al. (2016), Elnassen et al. (2016), Han et al. (2016), Cerquera Córdoba et al. (2017), O’Dowyer et al. (2017), Pessotti et al. (2018), Mandujano et al. (2020) y Ruisoto et al. (2020). 
Vol. 8 | Investigación Cualitativa en Salud: Avances y Desafíos

\section{Diagrama de Flujo de la selección de los estudios}

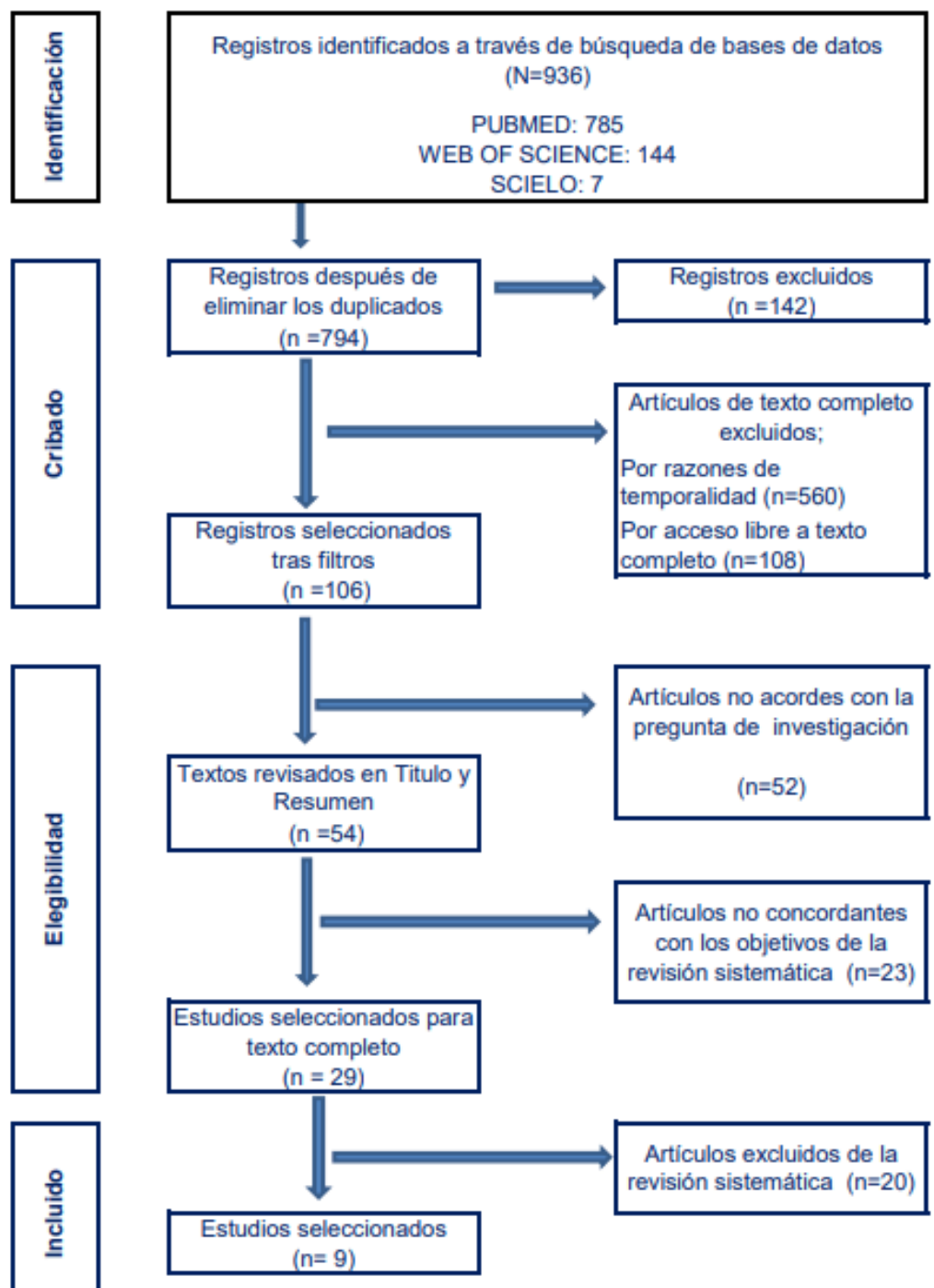

Fig. 3. Nota: Elaborado según PRISMA (2009).

\subsection{Evaluación de la calidad metodológica}

Los nueve artículos científicos finales resultantes se evaluaron mediante la escala de criterios PEDRo (Verghagen et al. 1998), empleada para categorizar la investigación con el fin de identificar la calidad de cada publicación (Maher, 2003). Está fundamentada en los criterios de la escala Delphi y el rango de puntuación va de 0 a 10 valorando 11 criterios. Las valoraciones comprendidas entre 9-10 puntos son consideradas una excelente calidad metodológica, de 6-8 puntos buena, de 4-5 aceptable y $<3$ como escasa calidad metodológica (Verhagen, 1998). Esta escala no evalúa la calidad externa del estudio sino la interna (Shiwa, 2017). A continuación, en la Tabla 2 se observa que los estudios de la revisión sistemática tienen una puntuación superior o igual a 7 , por lo que se evidencia una correcta calidad metodológica de los trabajos seleccionados. 
Vol. 8 | Investigación Cualitativa en Salud: Avances y Desafíos

Tabla 2. Nota: elaboración propia. Escala PeDro de artículos seleccionados

\begin{tabular}{|c|c|c|c|c|c|c|c|c|c|c|c|c|}
\hline Autores & 1 & 2 & 3 & 4 & 5 & 6 & 7 & 8 & 9 & 10 & 11 & Total \\
\hline $\begin{array}{l}\text { Jrapp } \\
\text { (2015) }\end{array}$ & SI & $\mathrm{SI}$ & SI & VO & $\mathrm{SI}$ & $\mathrm{SI}$ & $\mathrm{SI}$ & NO & NO & $\mathrm{SI}$ & $\mathrm{SI}$ & 8 \\
\hline $\begin{array}{l}\text { Dias, } \\
\text { (2016) }\end{array}$ & SI & $\mathrm{SI}$ & $\mathrm{SI}$ & SI & $\mathrm{SI}$ & $\mathrm{SI}$ & $\mathrm{SI}$ & $\mathrm{SI}$ & SI & NO & $\mathrm{SI}$ & 10 \\
\hline $\begin{array}{l}\text { Elnassen, } \\
(2016)\end{array}$ & $\mathrm{SI}$ & $\mathrm{SI}$ & $\mathrm{SI}$ & SI & $\mathrm{SI}$ & $\mathrm{SI}$ & $\mathrm{SI}$ & $\mathrm{SI}$ & SI & NO & $\mathrm{SI}$ & 10 \\
\hline Han, (2016) & $\mathrm{SI}$ & NO & $\mathrm{SI}$ & SI & $\mathrm{SI}$ & NO & $\mathrm{SI}$ & $\mathrm{SI}$ & SI & NO & $\mathrm{SI}$ & 9 \\
\hline $\begin{array}{l}\text { O'Dowyerc, } \\
(2017)\end{array}$ & $\mathrm{SI}$ & NO & $\mathrm{SI}$ & NO & $\mathrm{SI}$ & SI & $\mathrm{SI}$ & $\mathrm{SI}$ & SI & NO & $\mathrm{SI}$ & 8 \\
\hline $\begin{array}{l}\text { Resssotti. } \\
\text { (2018) }\end{array}$ & $\mathrm{SI}$ & $\mathrm{SI}$ & NO & SI & NO & NO & $\mathrm{SI}$ & NO & SI & $\mathrm{SI}$ & $\mathrm{SI}$ & 7 \\
\hline $\begin{array}{l}\text { Mandajano, } \\
(2020)\end{array}$ & $\mathrm{SI}$ & $\mathrm{SI}$ & $\mathrm{SI}$ & SI & $\mathrm{SI}$ & $\mathrm{SI}$ & $\mathrm{SI}$ & $\mathrm{SI}$ & SI & SI & $\mathrm{SI}$ & 10 \\
\hline
\end{tabular}

\section{Resultados}

De los artículos seleccionados encontramos 6 realizados en países latinoamericanos desde el año 2015 (Argentina y México), 2016 (Argentina/Brasil), 2017 (Colombia), 2018 (Brasil) y 2020 (México). Estos estudios tiene una horquilla de participantes de 10 (Colombia, 2017) a 130 (Argentina/Méjico, 2015), con un número total de 478. Estos muestran una correlación positiva significativa entre la resiliencia y calidad de vida del cuidador/a familiar, que sugiere que los cuidadores/as resilientes tienen una mejor percepción de vida. Por otro lado, si nos situamos en otros lugares del mundo como Australia (2017), EEUU (2019) y España (2020), los participantes oscilan de 21 a 283. En estos últimos estudios la resiliencia se conforma como un recurso individual del cuidador/a familiar innata o adquirida que evita la sobrecarga del cuidado sobre todo en la figura de la mujer cuidadora. A continuación se detallan los resultados en la Tabla 3.

Tabla 3. Descripción de los estudios seleccionados.

\begin{tabular}{|c|c|c|c|c|c|c|}
\hline Autor/fecha/país & Título del estudio & Tipo de estudio & Muestra & Objetivo & Tipo de intervención & Resultados \\
\hline $\begin{array}{l}\text { Trapp, S. K., Perrin, } \\
\text { P.B., Aggarwal, R., } \\
\text { Peralta, S. V., Stolfi, } \\
\text { M. E., Morelli, E., } \\
\text { Peña Obeso, L. A., \& } \\
\text { Arango-Lasprilla, } \\
\text { J.C., en } 2015 . \\
\text { Argentina y México. }\end{array}$ & $\begin{array}{l}\text { Fortalezas } \\
\text { personales y calidad } \\
\text { de vida relacionada } \\
\text { con la salud en los } \\
\text { cuidadores de } \\
\text { demencia } \\
\text { América }\end{array}$ & $\begin{array}{l}\text { Estudio } \\
\text { trasversal } \\
\text { descriptivo. }\end{array}$ & $\begin{array}{l}\text { Muestreo aleatorio de } 130 \\
\text { cuidadores/as familiares } \\
\text { entre ambos paises. }\end{array}$ & 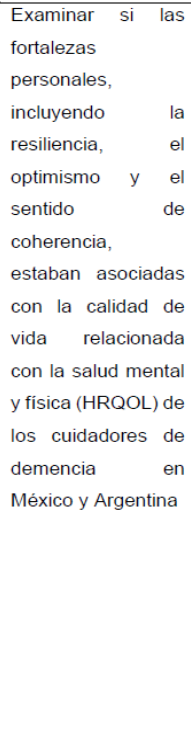 & 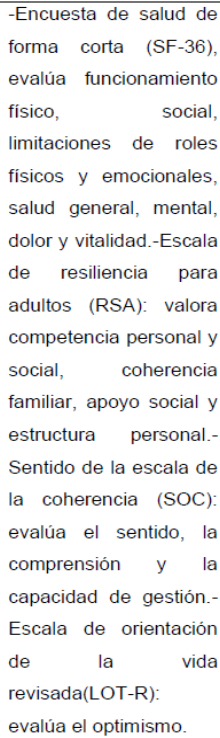 & $\begin{array}{l}\text { Los resultados son: las } \\
\text { fortalezas personales } \\
\text { (resiliencia, sentido de la } \\
\text { coherencia y optimismo) se } \\
\text { asocian en un } 58,4 \% \text { a la } \\
\text { calidad de vida mental } \\
\text { relacionada con la salud del } \\
\text { cuidador/a.- Las fortalezas } \\
\text { personales representan un } \\
8,9 \% \text { de la calidad de vida fisica } \\
\text { del cuidador/A familiar, y el } \\
\text { sentido de la coherencia es el } \\
\text { que se asocia más } \\
\text { significativamente a esta } \\
\text { salud.- Todas las fortalezas } \\
\text { están interrelacionadas entre si } \\
\text { y correlacionan tanto la calidad } \\
\text { de vida relacionada con la } \\
\text { salud fisica y mental del } \\
\text { cuidador/a familiar. }\end{array}$ \\
\hline
\end{tabular}




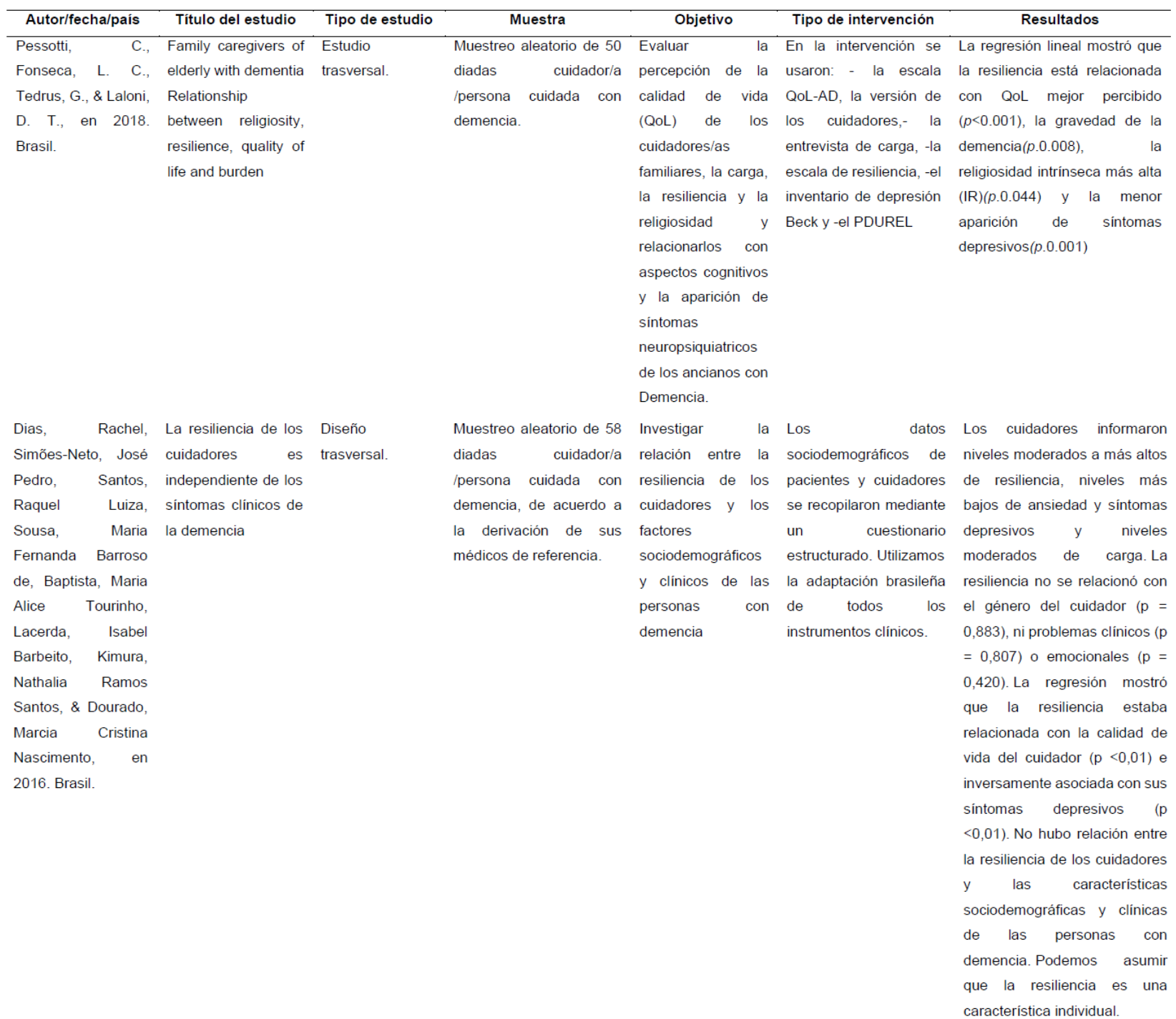

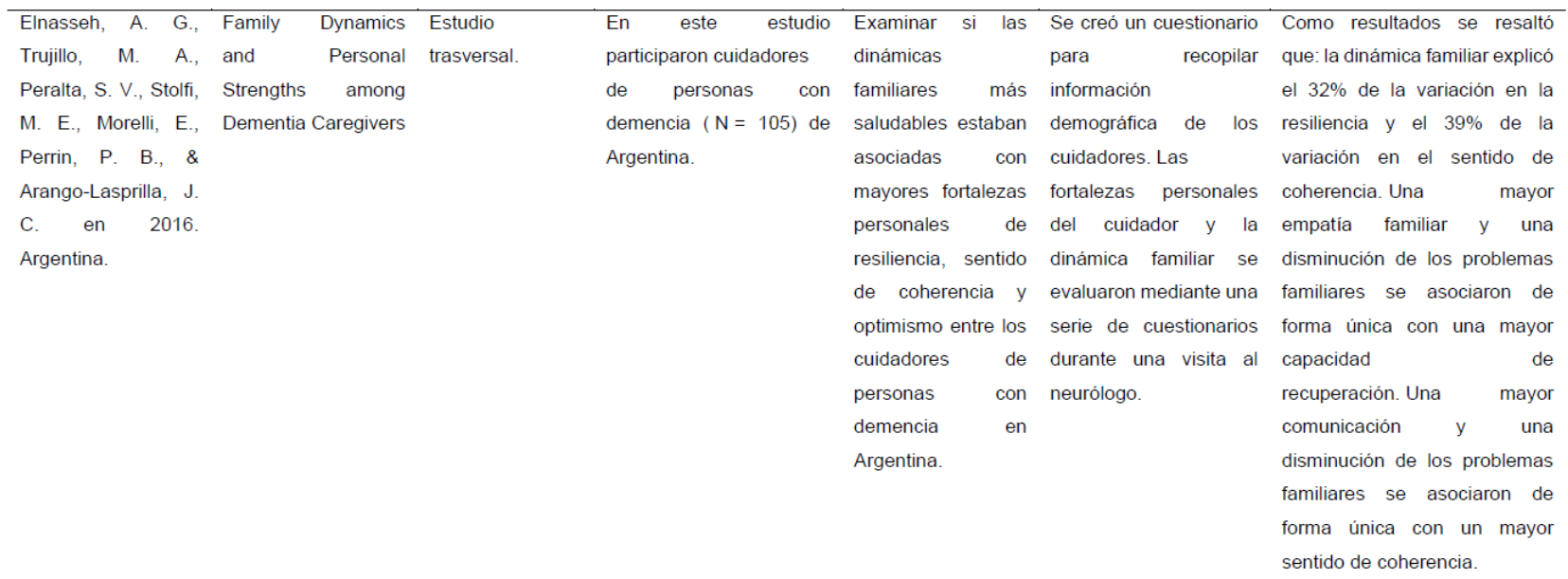




\begin{tabular}{|c|c|c|c|c|c|c|}
\hline Autor/fecha/país & Título del estudio & Tipo de estudio & Muestra & Objetivo & Tipo de intervención & Resultados \\
\hline $\begin{array}{l}\text { Han S, Chi NC, Han } \\
\text { C, Oliver DP, } \\
\text { Washington K, } \\
\text { Demiris } \text { G. en } 2019 . \\
\text { EEUU. }\end{array}$ & $\begin{array}{l}\text { Adapting the } \\
\text { Resilience } \\
\text { Framework for } \\
\text { Family Caregivers of } \\
\text { Hospice Patients } \\
\text { With Dementia. }\end{array}$ & $\begin{array}{l}\text { Estudio } \\
\text { trasversal. }\end{array}$ & $\begin{array}{l}\text { Se estudiaron } 39 \\
\text { cuidadores ( } 30 \text { mujeres; } 9 \\
\text { hombres con una edad } \\
\text { media de } 62 \text { años } \\
\text { [desviación estándar, DE: } \\
7,4] \text { ). }\end{array}$ & $\begin{array}{l}\text { Identificar los } \\
\text { desafís y las } \\
\text { posibles soluciones } \\
\text { que son recursos } \\
\text { para la resiliencia y } \\
\text { las consecuencias } \\
\text { esperadas desde la } \\
\text { perspectiva de los } \\
\text { cuidadores } \\
\text { familiares de } \\
\text { pacientes de } \\
\text { hospicio con } \\
\text { demencia. }\end{array}$ & $\begin{array}{l}\text { Los desafíos son: } \\
\text { dificultades en la } \\
\text { comunicación, la } \\
\text { prestación de atención y } \\
\text { la toma de decisiones, } \\
\text { la falta de conocimiento } \\
\text { y como desafios } \\
\text { emocionales: la } \\
\text { preocupación por la } \\
\text { selección del centro de } \\
\text { atención, la muerte con } \\
\text { dignidad y la falta de } \\
\text { conciencia pública. Los } \\
\text { recursos de resiliencia } \\
\text { se identificaron a nivel } \\
\text { individual, comunitario y } \\
\text { social. Los beneficios } \\
\text { son: la capacidad de } \\
\text { brindar una mejor } \\
\text { atención y tener una } \\
\text { mejor calidad de vida } \\
\text { tanto para los pacientes } \\
\text { como para los } \\
\text { cuidadores. }\end{array}$ & $\begin{array}{l}\text { Nuestros hallazgos muestran } \\
\text { que varios cuidadores } \\
\text { resilientes han encontrado y } \\
\text { utilizado recursos que pueden } \\
\text { ser informativos y útiles para } \\
\text { otros cuidadores. Su } \\
\text { experiencia puede informar las } \\
\text { intervenciones y crear } \\
\text { conciencia sobre la importancia } \\
\text { de proporcionar recursos de } \\
\text { resiliencia en múltiples niveles. }\end{array}$ \\
\hline $\begin{array}{l}\text { O'Dwyer, S. T., } \\
\text { Moyle, W., Taylor, } \\
\text { T., Creese, J., \& } \\
\text { Zimmer-Gembeck, } \\
\text { M., en } 2017 . \\
\text { Australia. }\end{array}$ & $\begin{array}{l}\text { In Their Own Words: } \\
\text { How Family Carers } \\
\text { of People with } \\
\text { Dementia } \\
\text { Understand } \\
\text { Resilience }\end{array}$ & $\begin{array}{l}\text { Estudio } \\
\text { trasversal } \\
\text { descriptivo. }\end{array}$ & $\begin{array}{l}\text { Muestreo de } \\
\text { conveniencia de } 61 \\
\text { cuidadores/as familiares } \\
\text { con una elección final de } \\
21 \text { participantes. }\end{array}$ & $\begin{array}{l}\text { Explorar las } \\
\text { definiciones de } \\
\text { resiliencia de los } \\
\text { cuidadores y sus } \\
\text { opiniones sobre los } \\
\text { factores asociados } \\
\text { con la resiliencia. }\end{array}$ & $\begin{array}{l}\text {-Encuesta trasversal } \\
\text { con entrevistas } \\
\text { semiestructuradas } \\
\text { centradas en la } \\
\text { resiliencia, el apoyo, los } \\
\text { sentimientos de suicidio } \\
\text { y de homicidio } \\
\text { asociados al cuidado. }\end{array}$ & $\begin{array}{l}\text { Los resultados se diferencian } \\
\text { por temáticas: } \\
\text { 1. La presencia de la } \\
\text { resiliencia: la mayoría de la } \\
\text { muestra evidencia su } \\
\text { existencia. } \\
\text { 2. El camino a la resiliencia: La } \\
\text { mitad de la muestra refiere que } \\
\text { la resiliencia es adquirida y una } \\
\text { necesidad. Una cuarta parte } \\
\text { subraya que es innata y refiere } \\
\text { que las normas culturales y de } \\
\text { género influyen en las } \\
\text { relaciones de cuidado y la } \\
\text { resiliencia. Por otro lado, la } \\
\text { suerte, el alto status económico } \\
\text { o los apoyos prácticos y } \\
\text { emocionales conllevan un } \\
\text { refuerzo de la resiliencia y un } \\
\text { sentimiento de agrado para } \\
\text { sobrevivir. } \\
\text { 3. Se evidencia que la falta de } \\
\text { algunas caracteristicas clave } \\
\text { dificulta la labor del cuidado. }\end{array}$ \\
\hline $\begin{array}{l}\text { Cerquera Córdoba, } \\
\text { A. M., Pabón, D. K., } \\
\text { \& Ruíz, A. L en } 2017 . \\
\text { Colombia. }\end{array}$ & $\begin{array}{lr}\text { Implementación del } & \text { de } \\
\text { programa } & \text { de } \\
\text { intervención } & \\
\text { psicológica } & \text { en } \\
\text { resiliencia } & \text { para } \\
\text { cuidadores } & \\
\text { informales } & \text { de } \\
\text { pacientes } & \text { con } \\
\text { demencia } & \text { tipo } \\
\text { Alzheimer PIRCA }\end{array}$ & $\begin{array}{l}\text { Se diseñó un } \\
\text { estudio } \\
\text { experimental } \\
\text { transversal } \\
\text { descriptivo } \\
\text { comparativo, tipo } \\
\text { pre-test/ post- } \\
\text { test. }\end{array}$ & $\begin{array}{lr}\text { Participaron } & 10 \\
\text { cuidadores } & \text { informales, } \\
\text { que no recibian } \\
\text { remuneración, } \\
\text { capacitación y } \\
\text { con el paciente. }\end{array}$ & 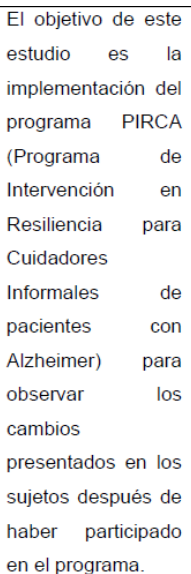 & $\begin{array}{l}\text { En la intervención se } \\
\text { usaron: programa } \\
\text { PIRCA (Programa de } \\
\text { Intervención en } \\
\text { Resiliencia para } \\
\text { Cuidadores Informales } \\
\text { de pacientes con } \\
\text { Alzheimer) }\end{array}$ & $\begin{array}{l}\text { Los resultados indicaron que } \\
\text { los puntajes de las variables } \\
\text { con mayor variabilidad fueron } \\
\text { depresión estado, eutimia } \\
\text { estado, sobrecarga, y } \\
\text { estrategias de afrontamiento } \\
\text { como búsqueda de apoyo } \\
\text { social y religión. Con lo que se } \\
\text { concluye que intervenir la } \\
\text { resiliencia responde a una } \\
\text { necesidad básica de los } \\
\text { cuidadores ya que está } \\
\text { presente en las características } \\
\text { de personalidad del cuidador. }\end{array}$ \\
\hline
\end{tabular}


Vol. 8 | Investigación Cualitativa en Salud: Avances y Desafíos

\begin{tabular}{|c|c|c|c|c|c|c|}
\hline Autor/fecha/pais & Título del estudio & Tipo de estudio & Muestra & Objetivo & Tipo de intervención & Resultados \\
\hline $\begin{array}{l}\text { Mandajano_Jaquez, } \\
\text { M., } \\
\text { Dominguez.Guedea, } \\
\text { M. T. \& Gaxiola- } \\
\text { Romero J.C. en } \\
2020 \text {. Mexico. }\end{array}$ & $\begin{array}{l}\text { Propiedades } \\
\text { psicométricas de una } \\
\text { escala de resiliencia } \\
\text { en cuidadores } \\
\text { familiares de adultos } \\
\text { mayores }\end{array}$ & $\begin{array}{l}\text { Estudio de tipo } \\
\text { transversal, } \\
\text { empírico, } \\
\text { cuantitativo, } \\
\text { instrumental, ya } \\
\text { que los datos de } \\
\text { los participantes } \\
\text { fueron } \\
\text { recolectados y } \\
\text { analizados en un } \\
\text { período de } \\
\text { tiempo particular } \\
\text { y además se } \\
\text { adecuaron y } \\
\text { estudiaron las } \\
\text { propiedades } \\
\text { psicométricas del } \\
\text { instrumento }\end{array}$ & 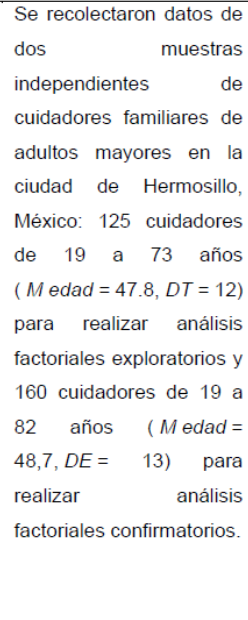 & $\begin{array}{l}\text { El objetivo de este } \\
\text { estudio fue probar } \\
\text { la validez de } \\
\text { constructo de un } \\
\text { inventario de } \\
\text { resiliencia } \\
\text { empleando } \\
\text { procedimientos } \\
\text { exploratorios y } \\
\text { confirmatorios. }\end{array}$ & $\begin{array}{l}\text { En la intervención se } \\
\text { usaron: en el RESI con } \\
12 \text { items: resiliencia } \\
\text { instrumental } \\
\text { resiliencia emocional }\end{array}$ & $\begin{array}{l}\text { Los análisis revelaron dos } \\
\text { dimensiones claras y } \\
\text { estadisticamente robustas en el } \\
\text { RESI con } 12 \text { ítems: resiliencia } \\
\text { instrumental y resiliencia } \\
\text { emocional. Este estudio } \\
\text { muestra que RESI es un } \\
\text { instrumento válido para evaluar } \\
\text { la resiliencia en una población } \\
\text { mexicana de cuidadores } \\
\text { familiares de adultos mayores }\end{array}$ \\
\hline $\begin{array}{l}\text { Ruisoto, P., } \\
\text { Contador, } \\
\text { Fernández-Calvo, } \\
\text { B., Serra, L., Jenaro, } \\
\text { C., Flores, N., } \\
\text { Ramos, F., \& Rivera- } \\
\text { Navarro, J. en } 2020 . \\
\text { España. }\end{array}$ & $\begin{array}{lr}\text { Efecto mediador del } \\
\text { apoyo social } & \text { en la } \\
\text { relación } & \text { entre } \\
\text { resiliencia } & \text { y } \\
\text { sobrecarga } & \text { en } \\
\text { cuidadores } & \text { de } \\
\text { personas } & \text { con } \\
\text { demencia } & \end{array}$ & $\begin{array}{l}\text { Estudio } \\
\text { trasversal } \\
\text { descriptivo. }\end{array}$ & $\begin{array}{l}\text { Se evaluó a un total de } \\
283 \text { cuidadores primarios } \\
\text { y familiares en España } \\
\text { para valorar las } \\
\text { características } \\
\text { sociodemográficas, el } \\
\text { estado clínico de las PCD } \\
\text { y las variables específicas } \\
\text { de los cuidadores y } \\
\text { cuidadores. }\end{array}$ & $\begin{array}{l}\text { Este estudio } \\
\text { examinó diferentes } \\
\text { factores predictivos } \\
\text { de sobrecarga en } \\
\text { una muestra de } \\
\text { cuidadores } \\
\text { familiares de } \\
\text { pacientes con } \\
\text { demencia } \\
\text { (PWD). En } \\
\text { particular, se probó } \\
\text { la influencia del } \\
\text { apoyo social y la } \\
\text { resiliencia sobre la } \\
\text { carga, } \\
\text { considerando los } \\
\text { posibles efectos de } \\
\text { la mediación. }\end{array}$ & $\begin{array}{l}\text {-Protocolo } \\
\text { estandarizado }\end{array}$ & $\begin{array}{l}\text { Los resultados avalan que el rol } \\
\text { de cuidador de personas con } \\
\text { discapacidad fue más común } \\
\text { en las mujeres, reportando } \\
\text { niveles de carga } \\
\text { significativamente más altos } \\
\text { que los hombres. La resiliencia } \\
\text { y el apoyo social explicaron la } \\
\text { mayor parte de la variación en } \\
\text { la carga. Además, el apoyo } \\
\text { social medió parcialmente la } \\
\text { relación entre resiliencia y } \\
\text { sobrecarga en los cuidadores. }\end{array}$ \\
\hline
\end{tabular}

\section{Discusión}

Lo que se puede extraer de los estudios revisados es que la resiliencia está presente en gran parte de los cuidadores/as familiares de una forma innata o adquirida (O'Dowyer, 2017) como fortaleza personal que aumenta la calidad de vida tanto física como mental del cuidador/a familiar (Trapp, 2015). Además, se asocia a unas características específicas (rasgos, comportamientos, recursos, valores y factores ambientales) intrínsecas en el cuidador/a familiar (O'Dowyer, 2017) evidenciándose que los cuidadores resilientes tienen menores síntomas depresivos, menor carga percibida y mayor calidad de vida (Pessotti, 2018). Por otro lado, se vincula un incremento de la resiliencia del cuidador/a familiar al factor de la suerte, un alto nivel adquisitivo y a las normas culturales y de género que predeterminan la mentalidad de la mujer cuidadora (O’Dowyer, 2017).

\section{Conclusiones}

El objetivo general de este trabajo fue analizar las consecuencias positivas del cuidado en el cuidador/a informal abordando la resiliencia como factor protector en la salud del mismo, que está al cuidado de personas mayores en situación de dependencia.

En este mismo sentido, la literatura revisada plantea que el cuidador/a informal resiliente aborda el cuidado con positividad y esto le genera bienestar y se plasman unas nociones básicas para abordar el cuidado con resiliencia similares a las características atribuibles al cuidador/a principal valoradas en la revisión sistemática. 
En definitiva, estas investigaciones son vitales dada la evolución de la población mayor hacia el envejecimiento y la dependencia, ya que los cuidadores/as informales son determinantes en su cuidado y su incremento es emergente. Por esta razón, es importante conocer los factores de protección de los que puede disponer el cuidador/a informal, para incidir sobre su salud y prevenir problemas futuros, además de potenciar unos cuidados de calidad a la población mayor dependiente de forma indirecta.

La investigación cualitativa da lugar a una reflexión sobre la resiliencia en el cuidador/a informal a través de un estudio que consigue los objetivos propuestos empleando teorías junto con unos métodos y técnicas cualitativas. Esta metodología da importancia y coherencia al factor protector de la resiliencia en el cuidador/a informal describiendo un conocimiento sobre la misma a través de dicha investigación.

\section{Agradecimientos}

A nuestro grupo de investigación HISAG-EP. Este estudio no ha sido financiado por ninguna entidad y no existen coflictos de intereses entre los autores.

\section{Referencias}

Araújo, C. L. \& Faro, A. C. (2016). Estudio sobre la resiliencia en ancianas del Valle de Paraíba, São Paulo, Brasil. Enfermería Global, 15(42), http://scielo.isciii.es/scielo.php?script=sci_arttext\&pid=S1695$61412016000200004 \& \operatorname{lng}=e s \& \operatorname{lng}=e s$.

Banchero, S. \& Mihoff, M. A. (2017). Personas mayores cuidadoras: sobrecarga y dimensión afectiva. Psicología, Conocimiento y Sociedad,7(1), 7-35. Epub 31 de mayo de 2017. http://www.scielo.edu.uy/scielo.php?script=sci_arttext\&pid=S1688$70262017000100007 \&$ Ing=es\&tlng=es

Beller, E.M., Glasziou, P.P., Altman, D.G., Hopewell, S., Bastian, H., Chalmers, I., Gøtzsche, P.C., Lasserson, T. \& Tovey, (2013). D. PRISMA for abstracts: Reporting systematic reviews in journal and conference abstracts. PLoS Med 10

Cerquera Córdoba, A. M., Pabón, D. K., \& Ruíz, A. L. (2017). Implementación del programa de intervención psicológica en resiliencia para cuidadores informales de pacientes con demencia tipo Alzheimer PIRCA. Universitas Psychologica, 16(2), 1-12. https://doi.org/10.11144/Ja veriana.upsy16-2.ipip

Dias, R., Simões-Neto, J. P., Santos, R. L., Sousa, M. F. B. de, Baptista, M. A.T., Lacerda, I. B., Kimura, N. R. S., \& Dourado, M. C. N. (2016). Caregivers' resilience is independent from the clinical symptoms of dementia. Arquivos de Neuro-Psiquiatria, 74(12), 967973. https://dx.doi.org/10.1590/0004-282x20160162

Ferreira, A.B.H. (1975). Novo dicionário da língua portuguesa. Rio de Janeiro: Nova Fronteira.

Elnasseh, A., Trujillo, M., Peralta, S., Stolfi, M., Morelli, E., Perrin, P. \& Arango-Lasprilla, J., (2016). Family Dynamics and Personal Strengths among Dementia Caregivers in Argentina. International Journal of Alzheimer's Disease. 2016. 1-10. 10.1155/2016/2386728.

Gálvez, A. (2004). Un ejemplo pedagógico: Cómo formular preguntas susceptibles de respuesta. Evidentia $2004 \quad$ ene-abr;1(1). En: http://cuiden.publicaciones.saludcastillayleon.es:80/evidentia/n1/4articulo.php [ISSN: 1697-638X].

Han, S., Chi, N. C., Han, C., Oliver, D. P., Washington, K., \& Demiris, G. (2019). Adapting the Resilience Framework for Family Caregivers of Hospice Patients With Dementia. American journal of Alzheimer's disease and other dementias, 34(6), 399-411. https://doi.org/10.1177/1533317519862095

Hedler, H.C., Faleiros, V. P., Santos, M. J. S., \& Almeida, M. A. A. (2016). Representación social del cuidador y cuidador familiar de los ancianos. Katálysis Magazine, 19(1), 143-153. doi: https://dx.doi.org/10.1590/1414-49802016.00100015.

Lindström, B. (2001). O significado de resiliencia. Adolescência Latino-Americana. 133-137. 
Lopes, C.C., Oliveira, G.A., Stigger, F.S. \& Lemos, A.T. (2020). Asociación entre la ocurrencia de dolor y sobrecarga en cuidadores primarios y el nivel de independencia del anciano en las actividades de la vida diaria: un estudio transversal. Cad Saúde Colet. https://doi.org/10.1590/1414$462 \times 202028010184$.

Machado, D., Silva, G., Bastos, A.E., Nazaré, M., Xavier, C. \& Souza, G. A., (2020). Experiencias subjetivas de vida de los cuidadores familiares de adultos mayores dependientes. Biblioteca Científica Electrónica Online. DOI: https://doi.org/10.1590/SciELOPreprints.1258

Maher, C.G., Sherrington, C., Herbert, R.D., Moseley, A.M. \& Elkins, M.M. (2003). Reliability of the PEDro scale for rating quality of randomized controlled trials.., 83. Phys Ther, 713-21

Mandujano Jaquez, M.F., Domínguez Guedea, M.T. \& Gaxiola Romero, J.C. (2020). Psychometric properties of a resilience scale in family caregivers of older adults. Acta Colombiana de Psicología, 23(2), 63-74. http://www.doi.org/10.14718/ ACP.2020.23.2.4

Martínez-Linares, J. M., Andújar-Afán, F. A., Martínez-Yébenes, R., \& López-Entrambasaguas, O. M. (2020). A Qualitative View of Homecare Support Workers on Unmet Health Needs of People with Dependency. International journal of environmental research and public health, 17(9), 3166. https://doi.org/10.3390/ijerph17093166

O'Dowyer, ST, Moyle, W., Taylor, T., Creese, J. y Zimmer-Gembeck, M. (2017). En sus propias palabras: cómo los cuidadores familiares de personas con demencia entienden la resiliencia. Ciencias del comportamiento (Basilea, Suiza) , 7 (3), 57. https://doi.org/10.3390/bs7030057

Organización para la Cooperación y el Desarrollo Económicos. Salud de un vistazo 2015: Indicadores de la OCDE. París: Publicación de la OCDE. doi: 10.1787/health_glance-2015-en.

Pereira, L. S., \& Soares, S. M. (2015). Fatores que influenciam a qualidade de vida do cuidador familiar do idoso com demência [Factors influencing the quality of life of family caregivers of the elderly with dementia]. Ciencia \& saude coletiva, 20(12), 3839-3851. https://doi.org/10.1590/1413 812320152012.15632014

Pesce, R. P., Assis, S. G., Avanci, J. Q., Santos, N.C., Malaquias, J. V. \& Carvalhaes, R. (2005). Adaptação transcultural, confiabilidade e validade da escala de resiliencia. Cad. Saúde Pública. Rio de Janeiro. 430-448.

Pessotti, C., Fonseca, L. C., Tedrus, G., \& Laloni, D. T. (2018). Family caregivers of elderly with dementia Relationship between religiosity, resilience, quality of life and burden. Dementia \& neuropsychologia, 12(4), 408-414. https://doi.org/10.1590/1980-57642018dn12-040011

Pinheiro, D.P.N. (2004). A resiliência em discussão. Psicologia em estudo, Maringá. 9, 1, 67-75.

Quero A., Briones R., Prieto M.a A., Pascual N., Navarro A. \& Guerrero C. Los cuidadores familiares en el Hospital Universitario de Traumatología y Rehabilitación de Granada. Index Enfermería 2005; 48/49:14-17

Ruisoto, P., Contador, I., Fernández-Calvo, B., Serra, L., Jenaro, C., Flores, N., Ramos, F., \& RiveraNavarro, J. (2020). Mediating effect of social support on the relationship between resilience and burden in caregivers of people with dementia. Archives of gerontology and geriatrics, 86, 103952. https://doi.org/10.1016/j.archger.2019.103952

Santillán, A. (2018). Cómo comenzar la búsqueda bibliográfica desde la pregunta PICO. Enfermería basada en la evidencia [blog]

Schmidt, A. E., llinca, S., Schulmann, K., Rodrigues, R., Principi, A., Barbabella, F., Sowa, A. et al., (2016) Fit for caring: factors associated with informal care provision by older caregivers with and without multimorbidity. European Journal of Ageing, 13(2), 103-113. doi: 10.1007/s10433-016-0373-4

Shiwa, S., Costa, L., Moser, A., de Carvalho Aguiar, I. \& de Oliveira, L. (2017). PEDro: la base de datos de evidencias en fisioterapia. Fisioterapia en movimiento, 24 (3). DOI: http://dx.doi.org/10.1590/S0103-51502011000300017

Trapp, S. K., Perrin, P. B., Aggarwal, R., Peralta, S. V., Stolfi, M. E., Morelli, E., Peña, L. A., et al. (2015). Personal strengths and health related quality of life in dementia caregivers from Latin America. Behavioural Neurology, 2015, 1-8. doi: 10.1155/2015/507196

Verhagen, A.P., de Vet, H.C.W., de Bie, R.A., Kessels, A.G.H., Boers, M., Bouter, L.M. \& Knipshcild, P.G.(1998) .The Delphi list: a criteria list for quality assessment of randomized clinical trials for conducting systematic reviews developed by Delphi consensus. , $51 \mathrm{~J}$ Clin Epidemiol. 1235-41

Whitbourne, S. K. \& Whitbourne, S. B. (2012). Demography of Aging. En S. K. Whitbourne \& M. J. Sliwinski (Eds.), The Wiley-Blackwell Handbook of 394 Adulthood and Aging. Oxford, England: WileyBlackwell. doi:10.1002/9781118392966.ch2 